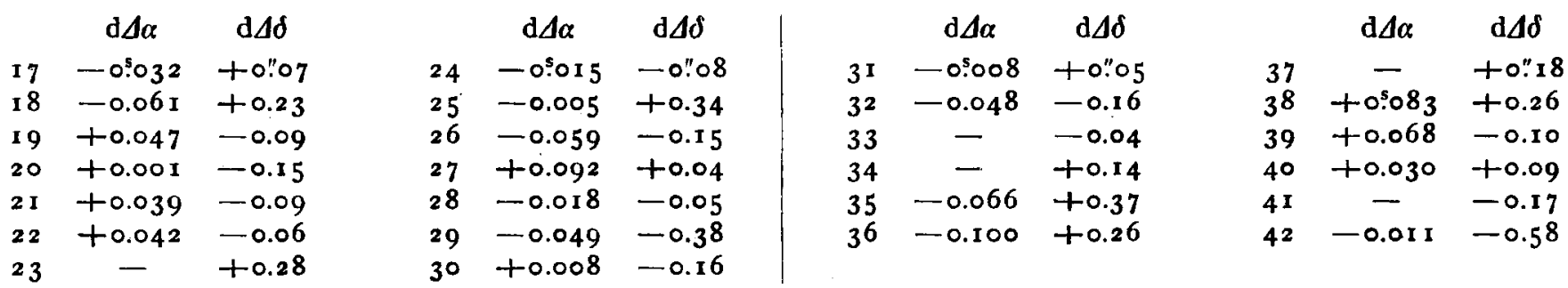

Die Uebereinstimmung der Rechnung mit den Beobachtungen ist eine befriedigende, und es liegt demnach kein Grund vor, statt der Kreisbahn elliptische Elemente für die Bewegung von Procyon zu berechnen.

Dorpat 1892 Mai.

L. Struve.

\title{
Observations de la Comète 1892 I
}

faites à l'Equatorial Brunner $(0.16 \mathrm{~m})$ de l'Observatoire de Lyon par M. G. Le Cadet.

\begin{tabular}{|c|c|c|c|c|c|c|c|c|c|c|c|c|c|c|c|c|c|c|c|c|}
\hline \multirow{2}{*}{\multicolumn{2}{|c|}{$\frac{1892}{\operatorname{Mars} 31}$}} & \multicolumn{3}{|c|}{ T. M. Paris } & \multicolumn{2}{|c|}{$\Delta \alpha$} & \multicolumn{2}{|c|}{$\Delta \delta$} & \multirow{2}{*}{$\frac{C p .}{3 \cdot 3}$} & \multicolumn{3}{|c|}{$\alpha$ app. } & \multirow{2}{*}{$\frac{\log p \cdot \Delta}{9.5 \circ 9 \mathrm{n}}$} & \multicolumn{3}{|c|}{$\delta$ app. } & \multirow{2}{*}{$\frac{\log p \cdot \Delta}{0.8_{30}}$} & \multicolumn{2}{|c|}{ Red. ad 1. app. } & \multirow{3}{*}{ - } \\
\hline & & & & & & 1.33 & $+5^{\circ}$ & 4.0 & & & & & & -7 & $7^{\circ} 3^{\circ}$ & & & -0.62 & - & \\
\hline \multirow[t]{11}{*}{ Avril } & I & 16 & 19 & 43 & $-I$ & & +8 & 21.2 & 4.2 & 20 & $5 I$ & & $9.529_{n}$ & -6 & 627 & $17 \cdot 1$ & 824 & $-0.6 \mathrm{I}$ & - & \\
\hline & I & 16 & 52 & 9 & -0 & 58. & +9 & 47. & 4.2 & & $5 \mathrm{I}$ & & & -6 & 625 & 50.7 & 29 & $-0.6 \mathrm{r}$ & -10.9 & \\
\hline & 3 & I 6 & 25 & $2 I$ & +3 & 0.17 & +13 & & & & 59 & & & -4 & 420 & & & -0.59 & & 3 \\
\hline & 4 & 16 & 24 & 53 & & & -7 & & 6.4 & & 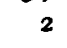 & & & -3 & $\begin{array}{ll}3 & 17\end{array}$ & & & & -1 & \\
\hline & 8 & & I I & 25 & $+\mathbf{I}$ & 2 & -4 & & 9.9 & 2 I & I 7 & & & +0 & - 49 & & & -0.58 & - & \\
\hline & 8 & I 6 & 40 & 7 & -0 & & $-\mathbf{I}$ & & 4.4 & & 17 & & & +0 & - 50 & & & & & \\
\hline & 9 & I 6 & 41 & 3 & $+I$ & & +17 & $I 8$ & 4.2 & $2 j$ & 2 I & & & +1 & I $5^{I}$ & 49.2 & & 7 &.$I$ & \\
\hline & I I & 16 & 2 & 18 & +0 & 40.50 & $-x_{4}$ & 31. & 4.2 & 2 I & 28 & & & +3 & 350 & .0 & & 57 & & \\
\hline & I & & 30 & 56 & +2 & $4 \cdot 3^{6}$ & +13 & 57 & 2.2 & 2 I & 42 & & & +7 & 743 & 10.0 & & 6 & $\cdot 3$ & \\
\hline & 2 & I 5 & 52 & 14 & -0 & 44. & -6 & & 6.8 & 22 & 9 & & & $+\mathrm{I}_{4}$ & 448 & & & -0 & & . \\
\hline & 27 & & 25 & 23 & +0 & & -0 & & 10.8 & & 2 I & & & +18 & I & & & & & \\
\hline \multirow[t]{9}{*}{ Mai } & 7 & I 4 & 42 & 11 & +0 & 46 & +6 & 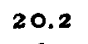 & 4.4 & & & & & & - & & & 0 & .9 & 12 \\
\hline & 9 & I 4 & 26 & 27 & $+\mathbf{I}$ & 17 & --3 & 56.9 & $4 \cdot 4$ & 20 & 57 & & & +26 & 620 & 49.6 & & 9 & & 13 \\
\hline & 9 & & Io & $3^{8}$ & +0 & 39 & +1 & I & 4.4 & 22 & 57 & & & & 621 & 59.0 & & 9 & & I \\
\hline & 10 & I 5 & 5 & $\mathbf{I}$ & +0 & 47 & -6 & & 4 & & & & $O_{n}$ & & 一 & & & $-0.4^{8}$ & & I \\
\hline & $I$ & I 5 & 24 & 39 & +3 & & -2 & 10.8 & 7 & 2 & & & & +27 & 734 & & & -0 & -12.8 & \\
\hline & . & I 4 & 50 & & -0 & $4 \cdot 4$ & +3 & & & & 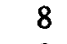 & & $5 n$ & +28 & 843 & & & -0.47 & -12.5 & 17 \\
\hline & I & I 5 & 23 & 22 & +0 & & -6 & 45 & 4.4 & 23 & 8 & & $17 \mathrm{n}$ & +28 & 844 & & & -0.47 & & $\mathbf{I}$ \\
\hline & 2 & I 4 & 59 & 6 & +0 & 32.38 & +0 & 9.9 & 4.4 & 23 & 43 & & & +35 & 540 & 27.6 & & -0.33 & $-11 \cdot 3$ & I \\
\hline & 30 & I 4 & 29 & I 5 & +0 & 11 & 一 IO & 50.6 & 4. & 23 & 50 & 59 & & $+3^{6}$ & $6 \quad 55$ & 7.9 & & -0.30 & 一 I I.I & $2 c$ \\
\hline \multirow[t]{3}{*}{ Juin } & 8 & 13 & I & 25 & +0 & 29.25 & -10 & 20.5 & 4.4 & o & 9 & & & +40 & 015 & 21.2 & & -0.14 & -10.1 & 21 \\
\hline & 9 & & $5^{\circ}$ & 57 & +0 & 34.7 I & -4 & $5^{8.7}$ & 4.4 & 0 & I I & & & +40 & - 35 & & 0.656 & -0.12 & -9.9 & 22 \\
\hline & 10 & I 2 & 25 & 35 & -0 & 7.70 & +4 & 49.9 & 4.4 & 0 & x 3 & 14.63 & $9.736_{n}$ & & $\circ 55$ & 18.2 & 0.707 & -0.10 & -9.8 & 23 \\
\hline
\end{tabular}

Positions moyennes des étoiles de comparajon pour I892.0.

\begin{tabular}{|c|c|c|c|c|c|c|c|c|}
\hline$*$ & \multicolumn{3}{|c|}{$a$} & \multicolumn{4}{|c|}{$\delta$} & Autorité \\
\hline $\mathbf{r}$ & $20^{\prime}$ & 49 & $\mathrm{n}_{17} \mathrm{~s}^{\mathrm{s}} 3$ & - & $7^{\circ}$ & $34^{\prime}$ & $56 " 8$ & $\begin{array}{l}1 / 3 \text { ( } 2 \text { Cord. GC. } 28689 \\
\quad+\text { Sj. } 8366)\end{array}$ \\
\hline 2 & 20 & 52 & 22.15 & $一$ & 6 & 35 & 27.4 & Sj. $84 \circ 3$ \\
\hline 3 & 20 & $5^{6}$ & 0.54 & - & 4 & 33 & 18.1 & Sj. $8445-6$ \\
\hline 4 & 21 & 3 & 2.69 & & 3 & 10 & 22.8 & $\begin{array}{l}1 / 2\left(\mathrm{Sj} .8514-5-6+\mathrm{M}_{1}\right. \\
2735 \mathrm{I})\end{array}$ \\
\hline 5 & $2 \mathbf{I}$ & 16 & 20.04 & + & $\circ$ & 54 & I 1.1 & $1 / 3\left(2 \mathrm{Gl} .543^{6}+\mathrm{M}_{1} 2814^{2}\right)$ \\
\hline 6 & 2 I & 18 & 25.40 & + & $\circ$ & & 5.1 & $\underset{28266)}{1 / 2\left(B B . V I+0.47 \times 9+M_{1}\right.}$ \\
\hline 7 & $2 \mathrm{I}$ & 20 & 1. $5^{8}$ & + & $\mathbf{r}$ & 34 & 42.6 & $\begin{array}{l}1 / 2\left(\mathrm{Sj} .8669-70+\mathrm{M}_{1}\right. \\
\left.\quad 28_{355}\right)\end{array}$ \\
\hline
\end{tabular}

\begin{tabular}{|c|c|c|c|c|c|c|c|}
\hline$*$ & \multicolumn{3}{|c|}{$\alpha$} & \multicolumn{3}{|c|}{$\delta$} & Autorité \\
\hline 8 & $2 \mathbf{I}^{1}$ & 27 & ${ }^{m} 45^{s} \cdot 31$ & $+4^{\circ}$ & 5 & - 8:7 & $1 / 2\left(M_{1} 28760+\right.$ GI. 5499) \\
\hline 9 & 2 I & 40 & 25.98 & +7 & 29 & 26.1 & $1 / 2\left(M_{1} 29435+\right.$ Gl. 5569$)$ \\
\hline 10 & 22 & 9 & $50.4 \mathrm{I}$ & +14 & 55 & 43.6 & Gl. $575^{2}$ \\
\hline I I & 22 & 21 & $33 \cdot 55$ & +18 & $\mathbf{I}$ & 56.1 & $W_{2} 22^{h} \cdot 432$ \\
\hline 12 & 22 & $5^{\circ}$ & 48 & +25 & $\circ$ & & BD. $+24^{\circ} 4683$ \\
\hline 13 & 22 & 55 & $5^{8.40}$ & +26 & 24 & 59.4 & $R \ddot{u}_{1}$ I0797 \\
\hline I 4 & 22 & $5^{6}$ & $41 \cdot 5^{\circ}$ & +26 & 20 & $5^{2} \cdot 3$ & $\begin{array}{c}\text { BD. }+26^{\circ} 455^{2} \text { rapportée } \\
\text { à } \operatorname{Ru}_{1} 10797\end{array}$ \\
\hline 15 & 22 & 59 & 20 & +27 & 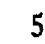 & & BD. $+26: 4559$ \\
\hline 16 & 22 & 59 & 53.18 & +27 & 37 & 9.4 & $W_{2} 22^{\mathrm{h}} \times 3^{26}-7$ \\
\hline I 7 & 23 & 8 & 25.74 & +28 & 40 & 37.6 & $W_{2} 23^{h} 107-8$ \\
\hline
\end{tabular}




\begin{tabular}{|c|c|c|c|}
\hline$*$ & $\alpha$ & $\delta$ & Autorité \\
\hline 18 & $23^{\mathrm{h}} \quad 7^{\mathrm{m}} 49^{\mathrm{s}} \cdot 7^{2}$ & $+28^{\circ} 5 I^{\prime} 22^{\prime \prime}$ & $W_{2} 23^{h} \cdot 95$ \\
\hline 19 & $\begin{array}{lll}23 & 43 & 9.86\end{array}$ & +354029. & $\mathrm{Arm}_{2} \mathbf{3 2 4 7}^{24}$ \\
\hline 20 & 2350 I9.7 I & 69.6 & $W_{2} 23^{h}$ IOI2-I 3 \\
\hline
\end{tabular}

\begin{tabular}{|c|c|c|c|}
\hline$*$ & $\alpha$ & $\delta$ & Autorité \\
\hline 2 I & $0^{\mathrm{h}} \quad 8^{\mathrm{m}} 54^{\mathrm{s}} 45$ & $+40^{\circ} 25^{\prime} 51.8$ & Gro. 24 \\
\hline 22 & ○ 1045.94 & +404041.7 & BB.VI $+40^{\circ} 4 \mathrm{I}$ \\
\hline 23 & o 1322.43 & $+405^{\circ} 3^{8.1}$ & $W_{2} 0^{h} \cdot 3 \circ 2-3$ \\
\hline
\end{tabular}

Remarques.

L'observation du 3 I Mars a été faite à l'Equatorial coudé $(0.36 \mathrm{~m})$ au moyen du micromètre à gros fils.

Mars 31. La comète se présente avec une condensation nucléaire à diamètre apparent de 6", entourée d'une nébulosité ronde de $10^{\circ}$ de diamètre environ, avec plusieurs prolongements filiformes et rectilignes que l'on distingue sur une longueur de plus de $10_{5}$ dans l'angle de position moyen $250^{\circ}$; elle apparait à l'oeil nu comme une petite nébuleuse allongée.

Avril I. Le noyau s'éteint dans la lumière crépusculaire en même temps que l'étoile de comparaison de $9^{\mathrm{e}}$ grandeur.

Avril 3. A l'œil nu et surtout par vision oblique, la comète apparait comme une étoile de $4^{\theta}$ grandeur dont la lumière serait diffusée par des cirrus, avec une queue trèspâle s'étendant rectilignement jusqu'à l'étoile de $4^{\mathrm{e}}$ grandeur, 3 Verseau, c'est à dire, sur une longueur d'environ $4^{\circ}$ dans l'angle de position $245^{\circ}$, opposé au soleil.

Avril $8_{(2)}$. Pendant les derniers pointés, la cornète éteinte par le crépuscule est à peine perceptible; on ne la revoit pas après la dernière lecture.

Avril 9. Des nuages amoncelés au Sud-Est retardent l'observation de la comete dont l'image est alors très-affaiblie par le crépuscule.

Dans ces observations, une comparaison comporte soit 5 pointés en $\Delta \delta$ et 5 pointés en $\Delta \alpha$; soit 5 pointés en $\Delta \delta$ et les passages à 3 fils. La colonne ${ } \mathrm{Cp}$ « donne le nombre de ces séries de pointés formant, pour chaque différence, autant d'observations complètes dont la moyenne est seule publiée. Ainsi, l'observation du 8 Avril résulte de 9 comparaisons complètes de la comète à l'étoile 5 faite dans des conditions très-différentes d'éclairement du champ ou des fils et qui, réduites au même instant au moyen du mouvement de la comète, donnent séparément pour comète-étoile :

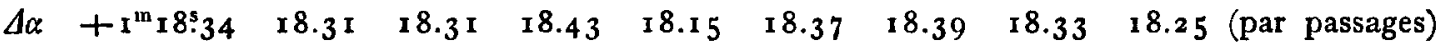

$$
\begin{aligned}
& \begin{array}{lllllllll}
\Delta d \quad-4^{\prime} 16.4 & 16.9 & 16.5 & 16.9 & 16.3 & 15.7 & 16.3 & 16.0 & 17.2
\end{array} \\
& \text { Moyenne }=-4^{\prime} 16.5 \text {. }
\end{aligned}
$$

Lyon (St. Genis Laval) le 21 Juin 1892.

\section{Observations of Wolf's Comet 1891 II and of Nova Aurigae.}

Observations of Wolf's Periodic Comet 189 II made at the Cambridge Observatory,

\begin{tabular}{|c|c|c|c|c|c|c|c|c|c|c|}
\hline 1891 & Berlin $M . T$. & \multicolumn{2}{|c|}{ Comet } & $\mathrm{Cp}_{\mathrm{p}}$. & \multicolumn{2}{|c|}{$\begin{array}{c}\text { Mean places of compared Stars } \\
\alpha 1891.0 \\
\delta 1891.0\end{array}$} & \multicolumn{4}{|c|}{ Authority } \\
\hline Aug. & 88 & $2^{\mathrm{h}} 5 \mathrm{I}^{\mathrm{m}} 54^{\mathrm{s}} \cdot 7^{2}$ & $+27^{\circ} 44^{\prime}$ I1:3 & 5 & $2^{\mathrm{h}} 49^{\mathrm{m}} 24^{\mathrm{s}} \cdot 23$ & $+27^{\circ} 48^{\prime} 53^{\prime \prime} 7$ & 4 & Mer. & Dbs. & nbridge \\
\hline & I 8.52635 & $\begin{array}{lll}2 & 59 & 45.09\end{array}$ & +272339.9 & 5 & $\begin{array}{lll}2 & 59 & 5.85\end{array}$ & +272641.7 & 3 & 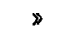 & 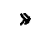 & 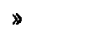 \\
\hline & 28.49743 & $\begin{array}{lll}3 & 24 & 27.97\end{array}$ & +254433.0 & 5 & $\begin{array}{lll}3 & 24 & 45.94\end{array}$ & +2547 & 4 & 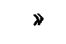 & » & $\gg$ \\
\hline & 28.49743 & $\begin{array}{lll}3 & 24 & 28.28\end{array}$ & +254434.0 & 5 & $\begin{array}{lll}3 & 26 & 0.96\end{array}$ & $+255^{\circ} 6.6$ & $\mathbf{I}$ & 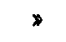 & 》 & 》 \\
\hline & 29.46946 & $\begin{array}{lll}3 & 26 & 45.27\end{array}$ & +253224.2 & 5 & $\begin{array}{lll}3 & 30 & 37 \cdot 3^{2}\end{array}$ & +253820.9 & 4 & $»$ & x & $\gg$ \\
\hline Sept. & 1.43745 & $3333^{8.76}$ & +245146.3 & Io & $334 \quad 15.63$ & $+245^{8} 35.5$ & 2 & » & $\gg$ & $\star$ \\
\hline
\end{tabular}
with the Northumberland Equatorial and Sqare Bar Micrometer. 